\title{
TECNOLOGIAS EM ALEITAMENTO MATERNO: REVISÃO INTEGRATIVA
}

\author{
Technologies in breastfeeding: an integrative review \\ Tecnologías para la lactancia materna: revisión integrativa
}

Artigo de Revisão

\section{RESUMO}

Objetivo: Identificar a produção científica existente na literatura quanto às tecnologias desenvolvidas para a promoção e incentivo à prática do aleitamento materno. Métodos: Revisão integrativa realizada nas bases de dados BDENF, LILACS, MEDLINE, PubMed. Inicialmente selecionados 103 artigos, sendo a amostra composta por oito artigos. A busca foi realizada nos meses de setembro e outubro de 2015 com os descritores: aleitamento materno (breastfeeding), tecnologias (technology), educação em saúde (health education). Resultados: Os artigos incluídos nesta revisão visaram avaliar a eficácia de tecnologias educativas como forma de incentivar a prática do aleitamento materno nas puérperas. Verificou-se que as tecnologias utilizadas foram álbum seriado, manual educativo, teleamamentação, CD-ROM, vídeo conferência e literatura de cordel. Conclusão: As tecnologias são uma estratégia para capacitação dos profissionais de saúde na assistência qualificada em aleitamento materno. $\mathrm{O}$ uso de tecnologias educativas implementadas na educação em saúde realizada pelos profissionais pode permitir impacto relevante na educação permanente para melhoria dos índices de aleitamento materno.

Descritores: Aleitamento Materno; Educação em Saúde; Promoção da Saúde; Tecnologias em Saúde.

\section{ABSTRACT}

Objective: To identify the scientific production in the literature regarding the technologies developed for promotion and encouragement of breastfeeding. Methods: Integrative review held in databases: BDENF, LILACS, MEDLINE, PubMed. It initially selected 103 articles, and the sample was composed or eight articles. The search was conducted in the months of September and October 2015 with the keywords: breastfeeding (aleitamento materno), technology (tecnologias), health education (educação em saúde). Results: The articles included in this review aimed to evaluate the effectiveness of educational technologies as a way to encourage the practice of breastfeeding in puerpera. It was found that the technologies used were flipchart, educational manual, online seminars, CD-ROM, video conferencing and Brazilian cordel literature. Conclusion: Technologies constitute a strategy for training health professionals in qualified care in breastfeeding. The use of educational technologies implemented in health education carried out by professionals, can allow significant impact on continuing education to improve breastfeeding rates.

Descriptors: Breastfeeding; Health education; Health Promotion; Health Technologies.
Anamaria Cavalcante e Silva ${ }^{(1,2)}$ Leilianne Maria Costa Freitas ${ }^{(2)}$ Josianne Alves de Freitas Maia ${ }^{(2)}$ Márcia Maria Ferreira Granja ${ }^{(2)}$ Regina Cláudia Melo Dodt ${ }^{(3)}$ Edna Maria Camelo Chaves ${ }^{(2)}$

1) Centro Universitário Christus Unichristus - Fortaleza - CE - Brasil

2) Universidade Estadual do Ceará - UECE - Fortaleza - CE - Brasil

3) Faculdade Metropolitana da Grande Fortaleza - FGF - Fortaleza - CE - Brasil

Recebido em: 18/05/2016

Revisado em: 14/07/2016

Aceito em: 20/08/2016 


\section{RESUMEN}

Objetivo: Identificar la producción cientifica de la literatura sobre las tecnologías desarrolladas para la promoción e incentivo a la práctica de la lactancia materna. Métodos: Revisión integrativa realizada en las bases de datos BDENF, LILACS, MEDLINE, PubMed. A principio fueron elegidos 103 artículos y la muestra final fue de ocho artículos. La búsqueda se realizó en los meses de septiembre y octubre de 2015 con los descriptores: lactancia materna (breastfeeding), tecnologías (technology), educación en promoción de la salud. Resultados: Los artículos incluidos en esta revisión eran aquellos que evaluaron la eficacia de las tecnologías educativas como incentivo a la práctica de la lactancia materna en las puérperas. Se verificó que las tecnologías utilizadas fueron el álbum seriado, el manual educativo, la tele lactancia, el CDROM, la videoconferencia y la literatura de cordel. Conclusión: Las tecnologías son estrategias para la capacitación de los profesionales sanitarios para la asistencia de calidad en lactancia materna. El uso de las tecnologías educativas en la educación en salud realizada por los profesionales puede permitir un impacto relevante en la educación permanente para la mejoría de los indices de lactancia materna.

Descriptores: Lactancia Materna; Educación en Salud; Promoción de la Salud; Tecnología Biomédica.

\section{INTRODUÇÃO}

O aleitamento materno até o sexto mês de vida proporciona muitos benefícios para o binômio mãe e filho, principalmente na redução da morbimortalidade infantil ${ }^{(1)}$, não se constituindo apenas em uma maneira de fornecer alimento e nutrientes. Amamentar também é acolher, cuidar, zelar, proteger, enfim, é uma infinita forma de amar para as mulheres que vivenciam esse momento.

Após o sexto mês o aleitamento materno é associado com alimentos complementares, que são importantes por apresentarem inúmeros benefícios nutricionais, imunológicos e afetivos, fundamentais para a saúde e sobrevivência das crianças. Esta prática é capaz de prevenir uma cada 7,5 mortes infantis, 50\% das mortes por doenças respiratórias e $66 \%$ daquelas causadas por diarreia ${ }^{(2,3)}$.

Diversos fatores comportamentais e psicológicos apresentados pelas mães podem ser encarados como preditores de sucesso ou insucesso da amamentação. Fatores tais como: mãe fumante, presença de sintomas depressivos, necessidade de retorno à escola ou ao trabalho, percepção errônea quanto à sua capacidade na produção do leite, relacionando o choro do bebê à fome, contribuem para o insucesso da amamentação ${ }^{(4)}$.

Amamentar é o ato de a mãe oferecer diretamente o seio, e o aleitamento materno é o meio pelo qual a criança recebe o leite de sua mãe, que pode ser pela amamentação por copo, por conta-gotas, por colher ou até mesmo por mamadeira. Amamentar é um processo de entrosamento entre dois indivíduos, um que dá e outro que recebe. A participação da família, em especial do pai, tem grande influência na amamentação ${ }^{(5)}$.

A promoção do aleitamento materno exclusivo até o $6^{\circ}$ mês de vida é a intervenção isolada em saúde pública que tem o maior potencial para a diminuição da mortalidade na infância ${ }^{(6)}$. A amamentação tem efeito protetor para a asma e infecções graves do trato respiratório inferior, reduzindo as chances de internação por pneumonia em lactentes ${ }^{(7)}$, assim como na redução de riscos para o desenvolvimento de doenças cardiovasculares e desenvolvimento da aterosclerose em adolescentes obesos ${ }^{(8)}$.

O colostro, primeiro leite produzido pela mãe, é nutritivo, contém anticorpos muitas vezes em quantidades maiores do que o leite considerado maduro, sendo suficiente e adequado para o bebê, mesmo em poucas quantidades. O leite materno é uma importante fonte de nutrição para o bebê, e tem em sua composição proteínas, gorduras e carboidratos, sendo o alimento essencial para o seu desenvolvimento. Possuem efeitos protetores contra doenças alérgicas, desnutrição, diabetes mellitus, dislipidemias, obesidade, cáries e hipertensão $\operatorname{arterial}^{(9)}$.

Estudo longitudinal realizado em uma maternidade mostrou que no momento da alta hospitalar, todas as mulheres amamentavam seus filhos, porém somente 98\% o faziam de forma exclusiva. Em relação ao aleitamento materno exclusivo no primeiro, quarto e sexto meses de vida, as prevalências foram $62,6 \%, 19,5 \%$, e $5,3 \%$, respectivamente ${ }^{(10,11)}$.

Segundo estudo realizado, $74 \%$ das mulheres indianas iniciam a amamentação no pós-parto, ainda na unidade hospitalar, porém em 3 meses apenas 35,7\% continuam amamentando exclusivamente seus filhos, e apenas $18,1 \%$ amamentam durante 6 meses de forma exclusiva ${ }^{(12)}$.

Na busca pela melhora dos índices de amamentação, muitas estratégias têm sido utilizadas nos serviços de saúde. Destacam-se nesse cenário as tecnologias como instrumento capaz de despertar o interesse dos usuários sobre a temática.

As tecnologias podem ser definidas como um conjunto de saberes e fazeres pertinentes a produtos e materiais que determinam terapêuticas e processos de trabalho e estabelecem instrumentos para realizar ações na produção da saúde ${ }^{(13)}$. No cenário de promoção ao aleitamento materno, nos deparamos com tecnologias utilizadas no processo de ensino e aprendizagem de gestantes e nutrizes que estão se preparando para vivenciar esse momento.

Este estudo teve como objetivo identificar a produção científica existente na literatura quanto às tecnologias 
desenvolvidas para a promoção e incentivo à prática do aleitamento materno.

\section{MÉTODOS}

Trata-se de uma revisão integrativa, método de pesquisa que sumariza resultados de pesquisas da literatura por meio de um processo de análise sistemática seguindo etapas: elaboração da questão de pesquisa; busca na literatura dos estudos primários com base nos critérios de inclusão e exclusão; organização dos estudos préselecionados (extração de dados dos estudos primários); análise crítica dos estudos primários selecionados; análise e síntese dos resultados avaliados; apresentação da revisão integrativa $^{(14,15)}$.

Para o desenvolvimento do estudo foi estabelecida a seguinte pergunta norteadora: "Quais as tecnologias que os profissionais de saúde têm desenvolvido para a promoção do aleitamento materno?" Os critérios de inclusão foram: artigos originais; temática compatível com o tema aleitamento materno associado ao desenvolvimento e uso de tecnologia; estar indexado nas bases de dados em inglês, português ou espanhol; estar disponível gratuitamente na íntegra; e data de publicação no período de 2010 a 2015. Os critérios de exclusão foram publicações duplicadas, relatos de experiências, editoriais, dissertações ou teses.

Para a busca dos estudos, utilizou-se consulta no DeCS e no MeSH com os seguintes descritores: aleitamento materno (breastfeeding), tecnologia (technology) e educação em promoção da saúde. Foram selecionados artigos escritos e ou publicados no período de 2010 a 2015 . A busca ocorreu no mês de setembro de 2015. As bases de dados utilizadas foram LILACS, MEDLINE, PubMed e BDENF.

Nas bases levantadas foram encontrados 32 artigos no PubMed, 27 artigos no LILACS, 36 no MEDLINE e 8 na BDENF com os descritores definidos. Após análise dos artigos, selecionaram-se 3 artigos no PubMed, 9 no LILACS, 17 no MEDLINE e 2 na BDENF. Foi realizada a leitura flutuante e com base nos critérios estabelecidos foram selecionados 6 no LILACS, 4 na MEDLINE, 2 na BDENF e 3 no PubMed em um total de 15 artigos. Dos 15 , quatro não tratavam de tecnologia, três eram comuns às bases, sendo selecionado apenas 1 de cada dupla, totalizando ao final 8 artigos (Quadro 1).

Para avaliação crítica, realizou-se a leitura na íntegra dos estudos e a releitura dos resultados, com finalidade de identificar aspectos relevantes que se repetiam ou se destacavam. Os artigos foram identificados por números, de acordo com a ordem de localização e organizados em ficha bibliográfica com os seguintes itens: autor, ano, tecnologia utilizada, revista, objetivo, resultados e conclusões.

\section{RESULTADOS}

Os artigos incluídos nesta revisão apresentam tecnologias educativas como forma de incentivar a prática do aleitamento materno nas puérperas, descrevendo as tecnologias educativas em saúde utilizadas para promoção do aleitamento materno, além de demonstrar sua eficácia na mudança de comportamento. Ressalta-se que o objetivo da educação em saúde vai além dá mudança de comportamento, busca-se o aprendizado por meio do desenvolvimento de outras práticas ${ }^{(16)}$. As tecnologias utilizadas para promoção do aleitamento materno visam favorecer a melhoria desses índices.

No quadro I encontram-se descritos o título, revista, ano, objetivo, resultados e conclusões dos artigos. Dentre os artigos selecionados 4 (50\%) foram escritos em 2013, 1 em 2011, 1 em 2012, 1 em 2014 e 1 em 2015.

A partir da análise dos objetivos propostos nos artigos, foi possível identificar que as produções visaram promover o aleitamento materno. Dois dos artigos utilizaram a literatura de cordel para favorecer a prática do aleitamento materno, além da construção e validação de manual educativo, álbum seriado, emprego da teleamamentação; CD-ROM e a videoconferência.

Verificou-se que a maioria dos estudos foi classificada como tecnologias leve-duras ${ }^{(25)}$ como álbum seriado, manual educativo, teleamamentação, CD-ROM, vídeo conferência e literatura de cordel.

Dos estudos selecionados, três foram estudos de validação, mostrando a importância desta etapa após a construção de materiais educativos.

\section{DISCUSSÃO}

O presente estudo traz contribuições no campo científico e prático, pois a divulgação dos estudos com tecnologias que possam contribuir para melhoria dos índices de amamentação são importantes para a saúde das crianças, que são vulneráveis aos agravos nos primeiros anos de vida. Como limitação, cita-se um número pequeno de estudos publicados com a utilização das tecnologias.

O tema aleitamento materno desperta sempre muito interesse na classe dos profissionais de saúde. Teoricamente aprende-se que amamentar é o melhor para a mãe e o bebê, pois são inúmeras as vantagens relacionadas com o leite materno. Na promoção do aleitamento, com o objetivo de facilitar o aprendizado para amamentação por parte das puérperas, várias tecnologias têm sido utilizadas como ferramenta.

Conceitua-se tecnologia como instrumento utilizado para promoção da saúde, constituída pelos saberes e 


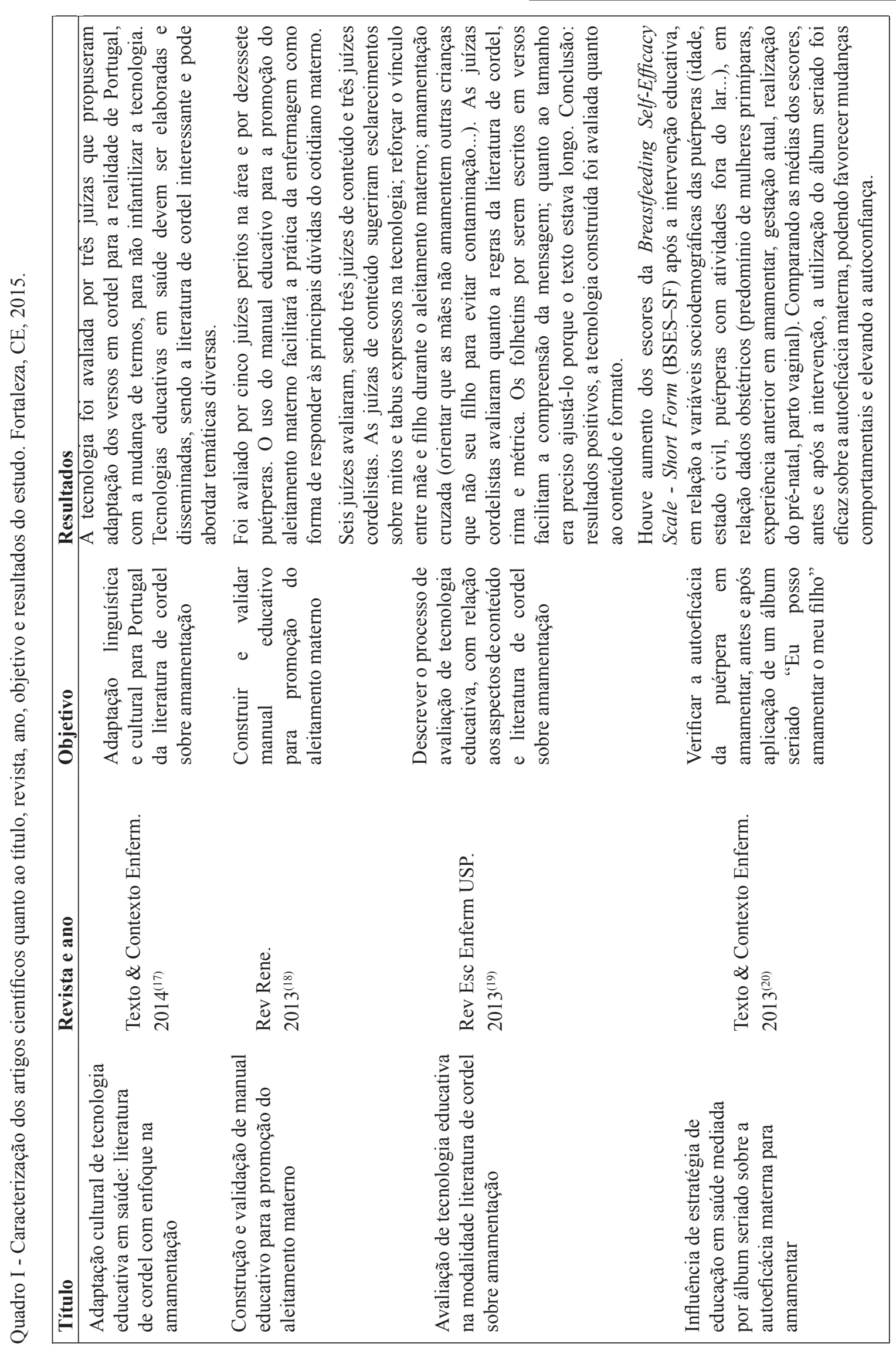




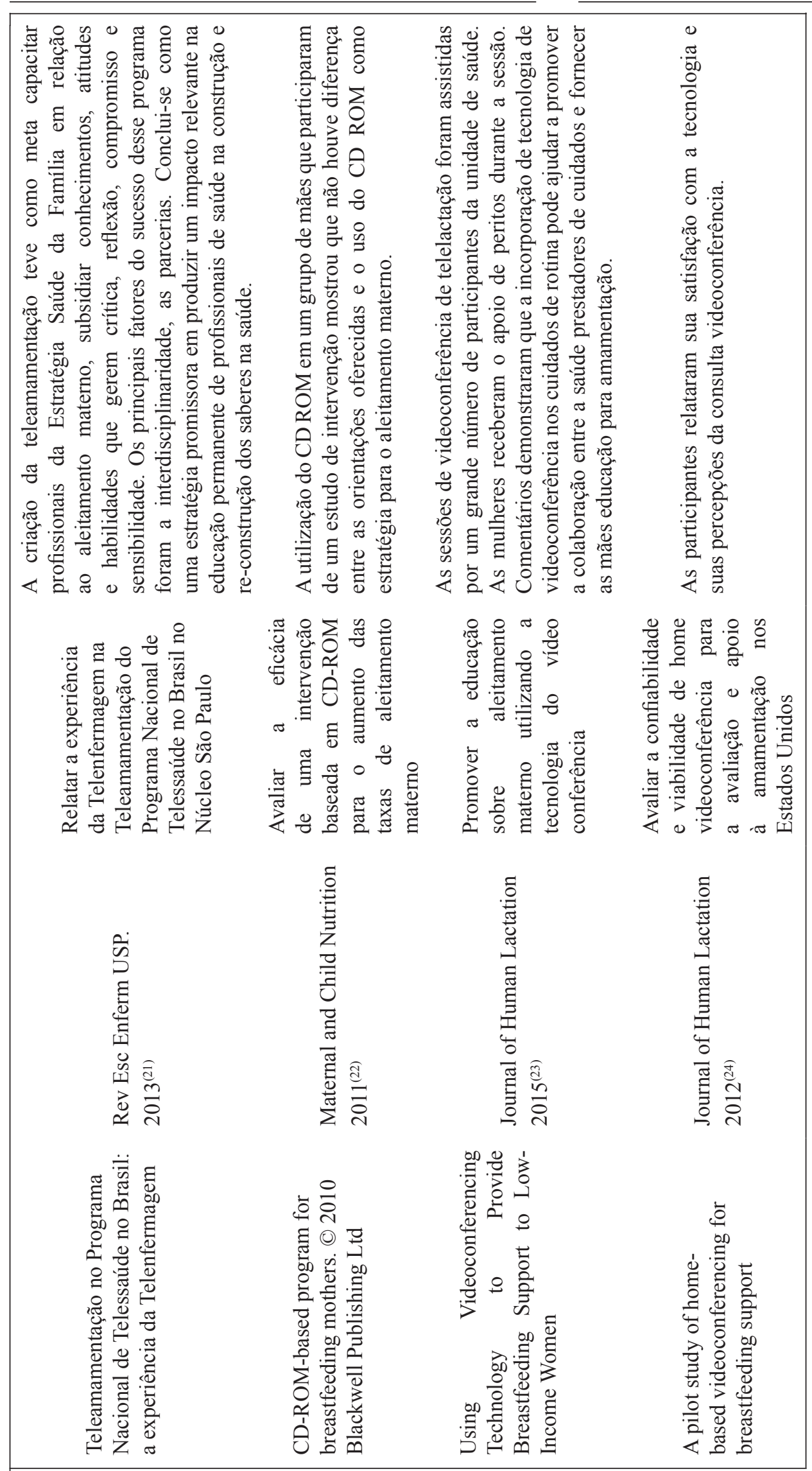


fazeres $^{(26)}$, com o objetivo de fortalecer a autonomia e capacidade de escolha dos indivíduos. $\mathrm{Na}$ prática assistencial, têm-se gestantes e nutrizes dentro desse processo.

A literatura aponta que a estratificação das tecnologias em saúde foram agrupadas em: tecnologia dura, tecnologia leve-dura e tecnologia leve, porém não se pode pensá-las de uma forma independente, elas estão interligadas ${ }^{(25)}$. A tecnologia não deve ser pensada como "máquinas" de fazer saúde e sim como formas de geração, produção e utilização de produtos para organização e melhoria das relações humanas ${ }^{(27)}$.

Todos os artigos selecionados nesta revisão integrativa utilizaram tecnologias leve-duras na promoção do aleitamento materno, realizada por profissionais ou estudantes, que buscavam sensibilizar as mulheres para a importância do ato de amamentar.

A literatura de cordel é uma forma de comunicação que retrata a cultura, o cotidiano, a realidade do povo e suas peculiaridades, podendo falar sobre qualquer assunto. É uma leitura que pode ser cantada ou declamada ${ }^{(28)}$. Sua origem remonta dos romances portugueses em versos, depois passados para a escrita, impressas em papel simples e penduradas em um barbante, daí o nome cordel ${ }^{(29)}$.

A literatura de cordel apresenta uma linguagem acessível, no entanto busca-se aliar o conhecimento científico aos saberes populares por meio desta tecnologia, com a elaboração da forma escrita e da forma cantarolada, tornando a tecnologia atrativa para o público alvo. Essa tecnologia deve ser adaptada aos costumes e realidades de cada localidade. A literatura de cordel com enfoque na amamentação, no Brasil, segundo o estudo realizado, mostrou a necessidade de elucidação de termos confusos e não apropriados e acréscimo de informações importantes para facilitar o entendimento. Esta tecnologia também foi utilizada com o mesmo objetivo em Portugal ${ }^{(30)}$.

Os manuais educativos são utilizados como estratégia de educação em saúde, devem ser fundamentados em termos científicos, e conterem propostas de atividades para recuperar e desenvolver aptidões físicas, mentais e sociais $^{(31)}$, uniformizando as orientações para facilitar o trabalho da equipe multidisciplinar com vistas ao cuidado em saúde ${ }^{(32)}$.

O manual educativo utilizado tanto no período prénatal e pós-natal facilita a prática da amamentação. Ao utilizar uma linguagem clara com ilustrações simples e compreensivas sobre aleitamento materno, desperta a atenção, dinamiza as ações e orientações de educação em saúde ${ }^{(33)}$. Para construção e validação do manual educativo, as autoras realizaram a validação de conteúdo e aparência por juízes especialistas e pelo público-alvo (puérperas) e concluíram que o manual era um instrumento pertinente e válido para os trabalhadores de saúde.

O álbum seriado é uma ferramenta utilizada nas unidades de saúde para a promoção em saúde e consiste em ilustrações e textos. Quando utilizado na unidade de saúde hospitalar logo após o parto, favorece a autoeficácia materna, sobretudo entre as puérperas de 20 a 29 anos, casadas ou que vivem em união consensual e que se encontram empregadas. Esse tipo de tecnologia é considerada leve-dura ${ }^{(25)}$.

$\mathrm{O}$ uso de tecnologia digital com o acesso a Internet tem permitido outras formas de divulgação do conhecimento. A Educação a Distância é uma ferramenta estratégica importante na qualificação dos profissionais para atuarem no mundo da informação, sendo uma tarefa complexa. O Programa Nacional de Telessaúde foi instituído no Brasil em 2007 com o "objetivo de desenvolver ações de apoio à assistência à saúde e, sobretudo, de educação permanente de Saúde da Família"(34), proporcionando aos profissionais, informações sobre amamentação nos diversos aspectos.

A tecnologia da videoconferência também pode oferecer orientações sobre aleitamento materno para as mães em sua própria residência, embora alguns problemas tenham sido identificados como baixa qualidade da conexão de Internet, som e imagens e variação dos tipos de computadores ${ }^{(35)}$.

A videoconferência é uma tecnologia inovadora que aumenta a acessibilidade ao serviço de saúde. Ela pode ser utilizada em tempo real, conectando consultores em lactação às mães que amamentam em seu próprio domicílio. Algumas limitações com a transmissão da tecnologia, como o áudio e vídeo podem comprometer o aleitamento materno e levar ao desmame precoce.

Para a viabilização das videoconferências, são necessários alguns requisitos tais como: disponibilização de recursos pelos gestores, conhecimentos prévios de informática, acesso otimizado à Internet, disponibilidade de computadores, além de tempo e horários disponíveis ${ }^{(36)}$.

A tecnologia de CD-ROM para a amamentação não adicionou benefícios às mães no pós-natal. Algumas causas possíveis para o insucesso dessa tecnologia devem-se ao fato de apenas $49,6 \%$ das mães matriculadas nas unidades, terem usado o CD-ROM $(0,8 \%)$, devido a dificuldades técnicas. O CD-ROM como estratégia para o aleitamento materno mostrou-se ineficaz como instrumento de tecnologia educativa.

As tecnologias utilizadas nos artigos desta revisão foram construídas a partir dos saberes estruturado dos profissionais de saúde, na busca da formulação de ações efetivas para a promoção ao aleitamento materno. Apesar de ser um tema vibrante com grande número de artigos escritos sobre o assunto, os artigos sobre a construção e a utilização de tecnologias na promoção do aleitamento materno ainda são poucos. 


\section{CONCLUSÃO}

As tecnologias apresentadas nessa revisão mostram que quando utilizadas contribuem para a melhoria dos índices de amamentação, sejam em particular as leve-duras e duras que foram as mais utilizadas nos estudos. A mudança de atitude das puérperas para a prática da amamentação passa pela mobilização afetiva, compreensão e interpretação cognitiva proporcionada pelo contato com a equipe de saúde.

A importância do uso destas tecnologias na assistência à amamentação constitui um importante instrumento que capacita os profissionais de saúde, destacando-se o uso do álbum seriado, a literatura de cordel, no entanto o acesso as informações digitais não é acessível para todos.

\section{REFERÊNCIAS}

1. Boccolini CS, Bocollini PMM. Relação entre aleitamento materno e internações por doenças diarreicas nas crianças com menos de um ano de vida nas capitais brasileiras e Distrito Federal, 2008. Epidemiol Serv Saúde. 2011;20(1):19-26.

2. Ramos CV, Almeida JAG, Saldiva SRDM, Pereira LMR, Alberto NSMC, Teles JBM, et al. Prevalência do aleitamento materno exclusivo e os fatores a ele associados em crianças nascidas nos hospitais amigos da criança de Teresina - Piauí. Epidemiol Serv Saúde. 2010;19(2):115-24.

3. Monte CMG, Giugliani ERJ. Recomendações para alimentação complementar da criança em aleitamento materno. J Pediatr (Rio J). 2004;80(5 Supl):S131-S141.

4. Queiroz AM, Silva FWGP, Borsatto MC, Nelson P Filho, Silva LAB, Díaz-Serrano KV. Inter-relação padrão de aleitamento e hábitos de sucção não nutritivos. Odontol. Clín.-Cient. Online. 2010 [acesso em 2015 Maio 29];9(3):209-14. Disponível em: http://revodonto.bvsalud.org/scielo.php?pid=S167738882010000300005\&script $=$ sci_arttext

5. Rego JD. O papel do pai na amamentação. In: Issler H. O aleitamento materno no contexto atual: políticas, práticas e bases científicas. São Paulo: SARVIER; 2008. v.1. p.17-23.

6. Toma TS, Rea MF. Benefícios da amamentação para a saúde da mulher e da criança: um ensaio sobre as evidências. Cad Saúde Pública. 2008; 24(Supl 2): 235-46.

7. Boccolini CS, Carvalho ML, Oliveira MIC, Boccolini PMM. O papel do aleitamento materno na redução das hospitalizações por pneumonia em crianças brasileiras menores de 1 ano. J Pediatr (Rio J). 2011;87(5): 399-404.

8. Masquio DCL, Piano A, Pereira CCA, Sanches PL, Campos RMS, Carnier J, et al. Aleitamento materno exclusivo atenua riscos cardiovasculares e estado inflamatório em adolescentes obesos. Alimentos Nutrição. 2014;25(1):33-9.

9. Costa LKO, Queiroz LLC, Queiroz RCCS, Ribeiro TSF, Fonseca MSS. Importância do aleitamento materno exclusivo: uma revisão sistemática da literatura. Rev Ciênc Saúde. 2013;15(1):39-46.

10. Ministério da Saúde (BR). DATASUS. Departamento de Informática do SUS. Brasília: Ministério da Saúde; 2012 [acesso em 2016 Abr 25]. Disponível em: http:// www2.datasus.gov.br/DATASUS/index.php

11. Chaves RG, Lamounier JA, César CC. Variáveis que afetam a duração do aleitamento materno. J Pediatr (Rio J). 2007; 83(3):241-6.

12. Centers for Disease Control and Prevention. Breastfeeding report card: United States 2014 [acesso em 2015 Maio 29]. Disponível em: http://www.cdc. gov/brestfeeding/pdf/2014brestfeedingreport.pdf. Published July 2014.

13. Nietsche EA. Tecnologia emancipatória: possibilidade para a práxis de enfermagem. Ijuí: UNIJUÍ; 2000.

14. Mendes KDS, Silveira RC CP, Galvão CM. Revisão integrativa: método de pesquisa para a incorporação de evidências na saúde e na enfermagem. Texto \& Contexto Enferm. 2008;17(4):758-64.

15. Souza MT, Silva MD, Carvalho R. Revisão integrativa: o que é e como fazer. Einstein. 2010;8(1):102-6.

16. Coscrato G, Pina JC, Mello DF. Utilização de atividades lúdicas na educação em saúde: uma revisão integrativa da literatura. Acta Paul Enferm. 2010; 23(2):257-63.

17. Oliveira PMP, Carvalho ALRF, Pagliuca LMF. Adaptação cultural de tecnologia educativa em saúde: literatura de cordel com enfoque na amamentação. Texto \& Contexto Enferm. 2014;23(1):134-41.

18. Costa PB, Chagas ACMA, Joventino ES, Dodt RCM, Oriá MOB, Ximenes LB. Construção e validação de manual educativo para a promoção do aleitamento materno. Rev RENE. 2013;14(6):1160-7.

19. Oliveira PMP, Pagliuca LMF. Avaliação de tecnologia educativa na modalidade literatura de cordel sobre amamentação. Rev Esc Enferm USP. 2013; 47(1): 205-12. 
20. Dodt RCM, Ferreira AMV, Nascimento LA, Macêdo AC, Joventino ES, Ximenes, LB. Influência de estratégia de educação em saúde mediada por álbum seriado sobre a autoeficácia materna para amamentar. Texto \& Contexto Enferm. 2013;22(3):610-8.

21. Prado C, Silva IA, Soares AVN, Aragaki IMM, Shimoda GT, Zaniboni VF, et al. Teleamamentação no programa nacional de telessaúde no Brasil: a experiência da telenfermagem. Rev Esc Enferm USP. 2013;47(4): 990-6.

22. José L, Nathalie GB, Laurent L, Domitille A, Camille S, Patrice F. CD-ROM-based program for breastfeeding mothers. Matern Child Nutr. 2011;7(30:263-72.

23. Friesen CA, Hormuth LJ, Petersen D, Tina Babbitt T. Using Videoconferencing technology to provide breastfeeding support to low-income women: connecting hospital-based lactation consultants with clients receiving care at a community health center. J Hum Lact. 2015;31(4):595-9.

24. Rojjanasrirat W, Nelson EL, Wambach KA. A pilot study of home-based videoconferencing for breastfeeding support. J Hum Lact. 2012;28(4):464-7.

25. Merhy EE. Em busca de ferramentas analisadoras das tecnologias em saúde: a informação e o dia a dia de um serviço, interrogando e gerindo trabalho em saúde. In: Merhy EE, Onoko R. Agir em saúde: um desafio para o público. $2^{a}$ ed. São Paulo: Hucitec; 2002. p. 113-50.

26. Joventino ES, Dodt RCM, Araujo TL, Cardoso MVLML, Silva VM, Ximenes LB. Tecnologias de enfermagem para promoção do aleitamento materno: revisão integrativa da literatura. Rev. Gaúcha Enferm. 2011;32(1):178-84.

27. Barra DCC, Nascimento ERP, Martins JJ, Albuquerque GL, Erdmann AL. Evolução histórica e impacto da tecnologia na área da saúde e da enfermagem. Rev Eletrônica Enferm [Internet]. 2006 [acesso em 2015 Junho 10];8(3):422-30. Disponível em: http://www. fen.ufg.br/revista/revista8_3/v8n3a13.htm.

28. Soares ACG, Tarifa AP, Marinho SAL. Os encantos da literatura de cordel. In: II Encontro PIBID/CAPES/ FAI. Ago 14-16; Adamantina, 2014. p. 1-4.

29. Alves RM. Literatura de cordel: por que e para que trabalhar em sala de aula. Rev Fórum Identidades. 2008;4:103-9.
30. Silva GRF, Cardoso MVLML. Material didático para a promoção da saúde ocular da criança. Rev Paul Enferm. 2007;26(1):12-6.

31. Gozzo TO, Lopes RR, Prado MAS, Cruz LAP, Almeida AM. Informações para a elaboração de um manual educativo destinado às mulheres com câncer de mama. Esc Anna Nery Rev Enferm. 2012;16 (2):306-11.

32. Echer IC. Elaboração de manuais de orientação para o cuidado em saúde. Rev Latinoam Enferm. 2005;13(5):754-7.

33. Oliveira MS, Fernandes AFC, Sawada NO. Manual educativo para o autocuidado da mulher mastectomizada: um estudo de validação. Texto \& Contexto Enferm. 2008;17(1):115-23.

34. Ministério da Saúde (BR), Programa Nacional de Telessaúde. Atenção Primária à Saúde: uma Ação Nacional de parceria entre os Ministérios da Saúde, Ciência e Tecnologia e Educação [Internet]. Brasília; 2010 [acesso em 2015 Ago 8]. Disponível em: http://www.telessaudebrasil.org.br/php/level. php?lang $=$ pt\& component $=42 \&$ item $=1$

35. Lindberg I, Christensson K, Ohrling K. Parents' experiences of using videoconferencing as a support in early discharge after childbirth. Midwifery. 2009;25(40:357-65.

36. Daza MPM, Berretin-Felix G, Machado MAMP. Requisitos para utilização de cybertutor com agentes comunitários de saúde. Rev CEFAC. 2014;16(2): $573-81$.

Endereço do primeiro autor:

Anamaria Cavalcante e Silva

Rua Ana Bilhar, 1163

Bairro: Varjota

60160-110 - Fortaleza - CE - Brasil

E-mail: anamariacasil@yahoo.com.br

\section{Endereço para correspondência:}

Edna Maria Camelo Chaves

Universidade Estadual do Ceará - UECE

Avenida Dr. Silas Muguba, 1700

Bairro: Itaperi

CEP: 60740-000 - Fortaleza - CE - Brasil

E-mail: ednacam3@hotmail.com 